\title{
Review on nano-drugs
}

\author{
Yong Liu ${ }^{1,3}$, Tian-Shui $\mathrm{Niu}^{2}$, Long Zhang1, Jian-She Yang ${ }^{1,2}$ \\ ${ }^{1}$ Key Laboratory for Natural Medicine, Lanzhou Institute of Chemical Physics, Chinese Academy of Sciences, Lanzhou, China \\ ${ }^{2}$ China Life Science College of Northwest Normal University, Lanzhou, China \\ ${ }^{3}$ Graduate School of Chinese Academy of Sciences, Beijing, China
}

Received 25 September 2009; revised 20 October 2009; accepted 22 October 2009.

\begin{abstract}
Nano materials is a new type of drug carriers with very promising application. In recent years, great progress was achieved in making drugs own the characteristics of targeted and controlled release via nanotechnologies. This paper addressed the capability of nano drugs on targeting to cells, penetrating through epicyte, controlled release and the security issues resulting from its using. We gave the prospect of nano drugs in biology and medicine applying.
\end{abstract}

Keywords: Nano Drug; Targeting; Cell Penetration; Controlled Release

\section{INTRODUCTION}

Nano drug is an important product of the rapidly developing nanotechnologies in biology and medicine field [1]. Drug is wraped in or adsorbed on surface of nanoparticles, when the specific targeting molecules combining with the receptor of cell surface, nano-drug is taken into cells, to achieve the safe and effective targeted drug delivery and gene therapy. Because Nano-drug carriers have high targeting, favorable sustained, controlled release capability and superior cell penetration ability, it can improve efficacy of drugs and reduce side effects. It is the production of nano-technology combining with modern medicine [2-3].

There are three main types of drug-loaded nanoparticles at the present time. First, the common drug-loaded particles: according to pharmacy technology bind with nanotechnology, with special methods to make the drug that physical-chemical property are unsteady and easy to be degradated or has considerable bad reaction to impact on the use highly dispersed in the drug carrier. Second, the controlled-release drug-loaded particles: be different from routine drug-loaded particles, this kind of drug's release process of nano-particles have a specific law. The dissolution of sac wall and the role of micro-organisms could make the drug in the heart of sac spread out. According to different purposes, choose a suitable tim- ber and technology to make particles gather on the local tissue and attaine effective concentration, without causing general toxicity reaction. Third, the targeting drugloaded particles: according to the needs of clinical, choose different carriers that have different affinity to different organizations or diseased region to make different carrier particles, or combine monoclonal antibody with the carrier, or under the effect of external magnetic field so that the drug can be transported to the particular site that we expected. Because nano-technology has changed the physical space of the drug, physicalchemical and biological property of drugs has surprising change. The changes mainly include the following aspects [2-7]: 1) Nanoscale drug carriers can enter into the capillaries, and freely flow in the blood circulation. It also can go through cells, be absorbed though pinocytosis by histiocyte and enhance bio-availability of drug; 2 ) Because the specific surface area of nano-drug carriers is very high, solubility of poor water-soluble drugs in the nano-carrier is relative enhanced and overcome the problems preparation with conventional methods; 3) Nano-carriers can be made to targeted position system, decrease the dose of drug and reduce the side effects with special processing. 4) By controlling the degradable speed of polymers in vivo, Nano-carriers can extend biological half-life of drug, improve the efficacy of the short-half-life drugs and reduce side effects of medication. 5) Because of Eliminateing the limit of specifial barriers such as blood-brain barrier, blood-ocular barrier and cell membrane barrier to the drug, nano-particulate drug carriers can pass through these barriers to treat scathing sites.

The central principle of nano-drug carrier is to realize drugs delivery "effective, safe and controllable". Therefore, targeting, controlled release and safety of drugs is an important and topical issues in pharmacy researchful area. The emergence of nano-delivery system make feasible to realize targeting and controlling release of drug. This paper expounds nano-drug delivery research in the field of medicine around the core.

\section{THE TARGETING OF NANO-DRUG}

Nano-drugs can selective distribute the object, to en- 
hance efficacy and reduce side effects. The role of the object from the target organ, target cell to the most advanced structure in the target cells. The three levels method of targeted therapy all could complete with nanotechnology. Nano-targeting drugs can be divided into passive targeting and active targeting.

\subsection{Passive Targeting}

Studies found that small particle size passive targeting drugs can spontaneously gather the diseased region making use of EPR (Enhanced Permeability and Retention) to achieve the purpose of passive targeting [8]. Because the blood capillary's permeability of the damage spot which caused by tumor, inflammation, hypertension and so on is higher than that of the normal blood vessel, simultaneous discharge capacity of lymph blood vessel is weaken. So in vivo long circulation the biological compatible macro-molecule, the medicine carrier, the molecular assembly are easier through blood vessel that injuries portion into the organization and assemble. The EPR is special useful to treat the tumor and blocks [9]. One of the ways to enhance EPR is strengthening the stability of drug to lengthen circulation time of drug in vivo. So the drug carriers have more opportunities to go through the target position and get together [10]. The passive targeting preparation include of micro-capsule, microsphere, nanopartiele, liposome and so on. The liquid crystal, fluid film, lipin, lipoid, protein and biodegradation high polymer material is often used as carrier material.

Mitra [11] studied the tumor targeting using dextran-doxorubicin-chitosan nanoparticles and showed that nanoparticles was not only reducing peripheral side effects, but also greatly improving the treatment of solid tumors. Du, et al. [12] made the carrier complex system with cyclic arginine-glycine-aspartic acid and lipid and combined interferon- $\alpha 1 b$ to treat liver fibrosis in rats, showed that according to combine with carrier, the concentration of interferon- $\alpha 1 b$ in the liver of rats was up to 10 times and the degree of liver fibrosis was significantly reduced comparing with non-carrier group. This illustrated the complex vector had a clear targeting to the liver. Briz [13-15] made two kinds of chelate compound with bile acid glycine-cisplatin and ursodeoxycholic acid-cisplatin, through the result of in vivo experiments showed that two complexes had a good affinity to the tumor cells of liver, and the absorbed dose was obvious higher than the original drug. Because of lower toxicity, these chelate compounds can extend more survival time of mice tumor transplant than the original drug. They also had effect to the chemical sproof tumor cells, and partly decrease physiological tolerance of tumor to the cisplatin.

\subsection{Active Targeting}

Active targeting is that drug carriers through the surface of nano-ligand binding specificity of targeting delivers drugs to specific organizations or release drugs in vivo under the certain physical conditions. The conventional active targeting mechanisms include three kinds. First, thermal-sensitive and $\mathrm{pH}$-sensitive targeting, that is, sufficiently use the changes of temperature and acidity that different body tissues and organs in the pathological process. Choose the polymer containing thermo-sensitive or $\mathrm{pH}$-sensitive (such as $\mathrm{N}$-isopropylacrylamide, etc.) component to form the polymer micelle. Drug-loaded micelles in the specific temperature or acidity can be easily depolymerized and released the drugs [16-18]. Thomas [19] reported a new type of temperature-sensitive nanoparticles. The critical solution temperature is $30^{\circ} \mathrm{C}$. The drug was wraped in the nanoparticles and the slow releaseing could last one month in vitro. When the temperature was more than $37^{\circ} \mathrm{C}$, the nanoparticles could priority be uptaken by the MDA2MB2231 breast cancer cells. This temperature-sensitive nanoparticles has great potential in the the treatment of thermal sensitivity targeting to the solid tumors.

$\mathrm{Na}$ [20] made PA-SDM nanoparticle with the amylopectin acetate (PA) and sulfanilamidesulfamidyl (SDM) that loadding adriamycin (ADR). The nanoparticles could change the rate of ADR release along with the alkalinity acidity change. As the $\mathrm{pH}$ value of the tumor spot was different from that of normal tissue, PA-SDM nanoparticles could selectively accumulate on the breast cancer cells MCF-7 and speed up the release, enhanced cytotoxicity to the tumour.

Yoo [21] got the $\mathrm{pH}$-sensitive polymer micelles complex by linking ADR with acid-sensitive. Taking advantage of meta-acid physiological characteristic of the tumor organization partial micro environment, adriamycin hydrolysis from the polymer micelles down when the drugs reached to the tumor site. Thereby enhanced the concentration of ADR in the tumor cells and increased efficacy of the drug.

These belong to the studies of the targeted drug delivery that in response to the environment, when the drug carriers meet with environmental stimulative, they are depolymerized to the monomers and drugs releases out of the vector. When combine EPR effect, nano-drug carriers that environment respond can further enhance the efficacy of antineoplastic.

Secondly, drug carriers can be modifed by combining with special targeting ligand (antibodies, lectins, sugars, hormones, etc.). Thereafter, this carrier-ligand complexity can be specifically identified by the epicyte receptors and accurately transmitted to the target spot.

Xiao [22] made the starch nanoparticles (StNP) charged negative electricity with reverse microemulsion and cross-linking methods, after StNP was modificed by a folic acid active substances (FA-PEG-NH ${ }_{2}$ ) modifiction, they successfully prepared the folic acid-starch 
nanoparticles (FA-PEG/StNP) which the average diameter was about $130 \mathrm{~nm}$. FA-PEG/StNP was combined with the anti-cancer drug doxorubicin (DOX) through penetration and got nano-drug containing folic acidstarch. Compared with StNP through hepatoma cells (BEL7404) culture experiments found that the cell lethality of using FA-PEG/StNP carrier was 3 times higher than that of StNP carrier. The result proved that FA was modified on the particles can significantly increased the particle targetting to the liver targeting cancer cells, made more drugs actting on the tumor cells and enhanced the drug's effect.

Jie [23] synthesized nanoparticles (NPs) of the blend of a component copolymer for targeted chemotherapy with paclitaxel used as model drug. The component was poly (lactide)-D-a-tocopheryl polyethylene glycol succinate (PLA-TPGS), which was of desired hydrophobic-lipophilic balance, which facilitates the folate conjugation for targeting. The nanoparticles were decorated by folate. The drugs were evidently promoted to targeting gather the surface of the breast cancer cells (MCF-7) and C6 glioma cells, thereby enhancing its efficacy.

Terada [24] established the specific targetting drug delivery system to the human hepatoma cell line (HCC). Through amino of dioleoyl phosphatidylethanolamine (DOPE) linked to substrate peptide of peginterferon matrix metalloproteinase- 2 that was modified by PEG and obtained PEG-PD, which could be enzymed cut by matrix metalloproteinase-2, then integrated the PEG-PD into the galactose-liposome and got the GaL-PEG-PDliposomes. Because the steric effect caused by PEG shielding the galactosyl of the surface of liposome complex, GaL-PEG-PD-liposomes could not be uptaken by the normal liver cells. But there was has high concentration of secreted matrix metalloproteinase- 2 around the HCC cells and could hydrolysis the peptide of GalPEG-PD-liposomes to remove the polyethylene glycol, relief the steric effects of polyethylene glycol, exposure the galactose residues of liposome surface. At this time the liposome could be recognised and uptaken by HCC cells and got the purpose of specific targeting to $\mathrm{HCC}$ cells.

Thirdly, suitable adjuvant was encapsulated into the micelles with physical method. The micellar will pulse release drug under the influence of the external excitation conditions (such as IR light, magnetic field). The adjuvant does not affect performance of micelles (stability, permeability, etc.), but impact the performance of the drug that is wrapped up in micellars (under certain conditions, hydrophilic can be converted to lipophilic, etc.). For example, Sershen [25] prepared N-isopropylacrylamide hydrogel could encapsulate $\gamma$-Fe2O3. Under the effect of outside magnetic field, when the temperature of hydrogel rised $10{ }^{\circ} \mathrm{C}$ and is higher than the critical solution temperature, hydrogel will rupture and sudden release the drugs.

Nanoparticles interacte with electromagnetic pulse or ultrasonic pulse can also enhance the release of drug. When the nanoparticles reach to the tumor vascular system and was adsorbed to the vessel wall, because electromagnetic pulse or ultrasonic pulse lead to the local thermal effects and further caused cavitation, tumor cell membrane is perforated, large molecular drugs enter into the cancer cells from blood, play the therapeutic effect.

\section{CELL PENETRABILITY OF NANO-DRUG}

There are many natural biological barriers to prevent the body suffering damage, such as blood brain barrier, blood-eye barrier, biomembrane barrier and so on, but the existence of these barriers also gives the difficulty to the treatment of morbidity spot. Nanoparticles is solid colloid particles that composed of macromolecule substance and the particlesize is $1 \sim 1000 \mathrm{~nm}$. It can pass various barriers. But as drug-carrier, if it can use its cell penetration and carries bioactive molecules into the targeting cell is the key problem of drug playing curative effect. In order to solve this problem, the researchers tested many sorts of nanomaterials. Yue [26] prepared nanometer sized-liposome that was produced from phosphatidylcholine to encapsulate fluorescent dyes $10^{-6}$ fluorescein isothiocyanate ihydrochloride (FITC), $10^{-6}$ Rhodamine B (RhoB). Liposome and fluorescent dyes was put into culture medium. After $2 \mathrm{~h}$, the result of confocal microscope screen showed that the FITC and Rho B couldn't go through cell membranes, fluorescence didn't exist in the cell, but green and red fluorescence were obserived in the liposomes groups. This explained that nano-liposomes could go into cell by cell endocytosis or fusion process, transfer fluorescent reagent that couldn't through membrane into cells. When the FITC-liposomes and liposomes Rho B coacted on cell, yellow fluorescence exited in cell, this account for liposomes containing different substances could into the cell at the same time.

According to Sivararnakrishnan [27] report, Betamethasone 17-valerat (BMV)-SIM had a good stability compared to traditional drug emulsion and skin absorption increased. In recycling experiments, the drug dose of skin containing was above $75 \%$.

Ding [28] prepared monostearin solid lipid nanoparticles (MSIN), investigated the cellular uptake of MSIN and the influence on the cellular uptake by MSIN modified with PEG2000 in human-type II cell alveolar epithelial cell line (A549) and murine macrophages cell line

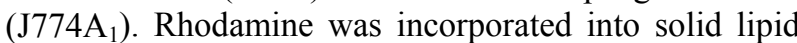
nanopartides as fluorescent marker. The experimental results showed MSIN that was modified with PEG2000 had low toxicity to cell and had good physiological compatibility. It was also highly taken by A549 cell line 
and could be fast reached to saturation. Pantarotto [29] prepared single walled carbon nanotubes modified by derivatization and single walled carbon nanotubes coupling peptides. They were all marked by FITC. Investigated the cellular uptake of the two-type functionalized carbon nanotubes (f2CNTs) and found that they all could penetrate the cell membrane: CNT1 mainly entered into the cytoplasm and the CNT2 that was modified by peptide could enter into the nucleus.

The studies also found that nano-materials that were uptaken by cells have the size critical point. Becker [30] prepared DNA-wrapped single-walled cabon and investigated length-dependent cellular uptaken of these carbon nanotubes. Studies showed that the cellular uptake of carbon nanotubes had a choice of lengths and the cut-off point was $(180 \pm 17) \mathrm{nm}$. They speculated that different cell might have different selective range of length to uptake the carbon nanotubes. Ito [31] used carbon nanotubes as EPO (erythropoietin, EPO) oral agent vector and found that the short carbon nanotubes could be used to carry more EPO to the target cells approved the speculation of Becker.

\section{THE CONTROLLABILITY OF NANO-DRUGS}

Nano-drug interactions with nano-carrier and made to be the controlled-release formulations with appropriate methods. When drug-carrier complex enter into the body, the drug is slowly released out of nanoparticles at the constant speed automatic in the scheduled time through the leaching, infiltration and proliferation or dissolution and act on the specific organ, tissue and cell. In addition, the nano-carriers prevent drug be degraded by various enzyme, extends the effective time of drugs. At the same time this controlled-release nano-drug can reduce the peak phenomenon of blood concentration, reduce side effects and improve efficacy. Mainly through diffusion control, chemical control, solvent control and other methods to achieve the purpose of controlled release of drug. Generally speaking, a controlled-release preparation has two or more controlled-release mechanisms.

\subsection{Diffusion-Controlled Release}

Drugs or other biologically active substances are combined with carriers; the drug is released in a certain of time and at a certain rate to the environment through diffusion. Diffusion-controlled is the most common mechanism in the controlled-release of drug delivery system, especially the nondegradable polymers carriers; the drug is mainly through this way released. In a biodegradable polymer carriers, when material degradation rate is slower than the diffusion of drug, diffusion of the drug still play a leading role in the release. There are many factors impact the diffusion-controlled release, such as geometric designs of system, condition and quality of ambient medium, the character and structure of the host materials, the solubility and loading amount of the drug [32].

\subsection{Chemical-Controlled Release}

Through hydrolysis, zymohydrolysis and other chemical reactions, chemical-controlled release system control the rate of drug release. According to the role of drug and substrate, mechanism of release, Chemical controlled system can be divided into degradable system and side-chain system. 1) Degradable system: the biological activity drugs is embedded or dispersed in biodegradable polymer, but there is no chemical bonding effects between drug and polymer, the rate of drug release is controlled by the rate of polymer degradation and erosion. The material of drug carrier is mainly include of biodegradable poly vinegar (such as polylactic acid, polycaprolactone with vinegar), poly polysaccharide (such as chitosan, gelatin), and so on. These materials is nontoxic, and the ultimate metabolites can be discharged in vitro or absorbed by organism, through regulating the rate of polymer degradation or dissolution to controll the release of drug on a specific location within regular hour. In these systems, the rate of polymer degradation or dissolution mainly influence the rate of drug's release, but the speed of degradation or dissolution also has an important relationship with the quality of the polymer (such as polymer molecular weight, crystallinity, the hydrophilic property and hydrophobicity, etc.), many researchers controlled and regulated the rate of degradation or dissolution material with chemical or physical methods such as reshaping, modification, blending to the polymer, further regulate the speed of drug release. But the nature of drug is also an important factor of the drug's release [33]. 2) The side-chain system of drug carrier may be degradable type or nondegradable type. Through the chemical bond that can be hydrolyzed or enzymolied, drugs in the side-chain system can be connected to the primary chain or side chain (side chain can be used to change the drug's release rate) of polymer. The release of drug is controllied through hydrolysis or enzymolysis.

Qing [34] used bovine serum albumin (BSA) as the model drug, at first, the nanoparticles containing proteins were obtained by absorbing BSA from the solution onto the surface of nano-scale $\mathrm{SiO} 2$, then, PLGA microsphere loading the solid nanoparticles were fabricated with the solid-in-oil-in-water-emulsion method. Study found with the increasing of the mass fraction of BSA in the product of adsorption, the rate of solvent controlled release of BSA is faster. The main mechanism of drug release is the diffusion of drugs and the degradation of the polymer. In the release process, the BSA that was on the surface of microspheres was first diffused and 
formed pores. It was conducive to the diffusion of the BSA in the inner layer. Water also could go into the microspheres and resulted in the degradation of microspheres. Microspheres that loaded more drugs diffused more BSA in the early period and also the pores that formed were more and larger, the degradation of the microspheres is faster in the late period, so release of BSA was faster.

Yang [35] prepared microspheres that containing antiphthisic drug Rifampin was prepared from poly lactic-co-glycolic acid (PLGA) as carrier by emulsion and solvent evaporation method. In vitro experiment of release, investigated the performance of PLGA microspheres that was as a carrier of drug delivery. The release time of rifampicin in the PLGA microspheres was more than 30 days, and there was no obvious phenomenon of sudden release. But the release of the mass fraction of rifampicin without microspheroidization was up to $96 \%$ in 10 minutes. At the same time, they found PLGA molecular weight and the LLA / GA mass ratio had significant impact on the time of the release [36,37]. Because the rate of degradation of low molecular weight PLGA was significantly higher than that of high molecular weight and in the PLGA copolymer, with the GA mass increaseing, hydrophilicity of PLGA enhanced, the degradation significantly speed up. While the rate of the drug's release was mainly controlled by the degradable rate, so with the reducing of PLGA molecular weight and LLA/GA mass ratio, the release of rifampicin speed up. The drug release was simultaneously controlled by drug-diffusion and degradation of carrier material, but in this system, degradation played a decisive role in the mechanism of control. Observed the surface morphology of the degradation rifampicin-PLGA microspheres, they found the surface and inside of microspheres appeared large holes, spherical shape almost disappeared, the red faded and turned to white. These result showed that in early period the drug's release was out of carrier materials only through the drug's diffusion and dissolution, with the drug release time prolonging, the mass fraction of the unit of drugs in microspheres reduced, lead to the release rate of drug that diffusion and degradation decrease, however, with the carrier material degrading and the rate of degradation speeding up, the primarily release of drug was degradation of materials, and made up the rate of diffusion and dissolution release reducing, eventually led to the drug in microsphere carrier was release in a constant velocity.

Solvent-control include of infiltration and swelling mechanisms. 1) The release of solvent infiltration controlled. It accord to the penetration principle of semipermeable membrane. Soluble drug is wrapped in polymer, when it is added in environmental media, the external solvent go into polymer matrix by infiltration and forms saturated solution and then under the action of osmotic pressure between saturated solution and envi- ronmental media to release drugs outside. 2) Matrix solvent control, the more common mechanism is swelling. The controlled-release mechanism is using solvent penetration to makes polymer swelling and achieve the purpose of release. At the beginning, solvent penetrate into polymer matrix and cause to swelling, the polymer glass transition temperature to the environment, and chemical chain get slack, so that drugs can be released. Solvation process often contains the spread process of drugs at the same time.

The release of drug is affected by many factors and conditions, including nature of polymer and drugs, temperature of environment, $\mathrm{pH}$ value of medium and so on. The change of one factor or condition will affect the controlled mechanism of drug's release. For example, changing the hydrophilicity and hydrophobicity of degradable polymer not only affect the rate of degradation of materials, but also affect swelling and permeability of the material, further affect the release of drugs.

Wang [38] prepared self-assembled nanomicelle of $\mathrm{N}$-acylcholesteryl succinate-O-carboxymethyl chitosan, paclitaxel was used as a model drug. In vitro experiment, they found that release rate of paclitaxel in nano-CCMC micelle was closely related with the $\mathrm{pH}$ value. For example the rate of release is low when the $\mathrm{pH}$ value of PBS was equal to 7.2, but when $\mathrm{pH}$ value was equal to 4.0 or 9.0 , the release rate increased. Because CCMC molecules were a new type of polymer ampholyte and containing much free- $\mathrm{NH} 2$ and-COOH, the isoelectric point was about 7.14 by the turbidity method detection [39]. In meta-acid or alkaline solution, the free- $\mathrm{NH}_{2}$ or- $\mathrm{COOH}$ in $\mathrm{CCMC}$ molecules was ionized to $-\mathrm{NH}^{+3}$ or-COO${ }^{-}$. Under the action of charges with the same electrical sign repel each other; the gel network structure of self-assembled nano-micelles (CCMC) fully absorbed water, increased permeability of paclitaxel, accelerated the release rate.

\section{THE SAFETY OF NANO-DRUG}

Accordance to the interpretation of ISO meeting [40]: biocompatibility means that the capability that the lives tissue has a reaction to inactive materials. Generally refers to the compatibility between materials and host, including histocompatibility and blood compatibility. Nano-bio-medical materials not only has the long-term stable physical and mechanical properties in the biological conditions, but also has side effect to the organization, blood, immune system, etc, that is, non-toxic, tissue compatibility, blood compatibility and so on. At the same time biocompatibility is generally considered to have two major principles, one is the principle of 'biosafety' and the second is the principle of 'biofunctionality' (or the effect of promoting the function to the body). Nano-biological materials are a foreign body for 
the host, so there will be some sort of response or repulsive phenomenon in the body. If nano-biological materials could be applied successfully, at least, the response that it caused should be acceptable by host, and shouldn't make harmful effects. So the biological materials should be carried on the evaluation of the nanobio-safety, that is, the biological evaluation. This is also the key tache that if nano-biological material can enter to clinical research [41]. So research about this area is active. As early as 1999, Richardson, etc. [42] had been studied the distribution of chitosan gene carriers in vivo. Highly purified chitosan fractions of $<5000 \mathrm{Da}, 5000$ $10000 \mathrm{Da}$ and $>10000$ Daltons were prepared and characterised in respect of their cytotoxicity, ability to cause haemolysis, ability to complex DNA as well as to protect DNA from nuclease degradation. The observations that the highly purified chitosan fractions used were neither toxic nor haemolytic, that they have the ability to complex DNA and protect against nuclease degradation and that low molecular weight chitosan can be administered intravenously without liver accumulation suggest there is potential to investigate further low molecular weight chitosans as components of a synthetic gene delivery system. Kim [43] synthesized biocompatible silicaovercoated magnetic nanoparticles containing rhodamine $\mathrm{B}$ isothiocyanate (RITC) within a silica shell of controllable thickness [MNPs@SiO2(RITC)]. In that study, the MNPs@SiO2(RITC) with 50-nm thickness were used as a model nanomaterial. After intraperitoneal administration of MNPs@SiO2(RITC) for 4 weeks into mice, the nanoparticles were detected in the brain, indicating that such nanosized materials can penetrate blood-brain barrier $(\mathrm{BBB})$ without disturbing its function or producing apparent toxicity. After a 4-week observation, MNPs @ $\mathrm{SiO} 2$ (RITC) was still present in various organs without causing apparent toxicity. Through labeling with rhodamine and got 50nm MNPs@SiO2 (RITC). Taken together, they demonstrated that magnetic nanoparticles of 50-nm size did not cause apparent toxicity under the experimental conditions of this study.

Yang [44] studied the distribution and toxicity of silicon nanoparticles in vivo. When silica nanoparticles suspension injected in mice, after $96 \mathrm{~h}$, electron microscopy result showed that silica nanoparticles distributed in the brain, liver, heart, spleen, lung, kidney, stomach, intestines, prostate, testis and other organs, and found a large quantity of silica nanoparticles had enter into the cell nucleus of liver and a small amount that cell nucleus of brain. Gave $4500 \mathrm{ug} / \mathrm{kg}$ nanoparticles to mice through intraperitoneal injection, two weeks later, there was no obvious abnormalities among mice body weight, appetite, defecation when compared to the control group, none was dead. But some scholars also believe that when the particle size reduced to a certain degree, the substance and material that original have non-toxic or low toxic begin to appear toxicity or toxicity significantly strengthened; and nano-materials may cause special situation of metabolism, have special toxicity.

Lam et al. [45] studied toxicity of carbon nanotubes to the organisms. Compared with carbon black and quartz (pink), carbon nanotubes $(0.1-0.5 \mathrm{mg} / \mathrm{kg})$ were injected into rats through trachea. The result showed that the group of carbon black rats was normal, the group of quartz rats had mild to moderate inflammation, observed lung epithelial granuloma in carbon nanotubes group and had relationship with dose-response. These results show that, if carbon nanotubes reach the lungs, they are much more toxic than carbon black and can be more toxic than quartz, which is considered a serious occupational health hazard in chronic inhalation exposures. Service [46] used polytetrafluorethylene (PTFE)-nano to do the inhalation contamination experiment on rat, the diameter of PTFE-nano was $20 \mathrm{~nm}$, the rats were contaminated 15 min, the majority of rats died within $4 \mathrm{~h}$, but rats would not be affected when the diameter was $130 \mathrm{~nm}$. Yang [47] studied the distribution of the original single-walled carbon nanotubes in rats. They found carbon nanotubes mainly distributed in the liver, lungs and spleen, and had long residual time, minute quantity was excluded outer the body through urine or feces. Though hadn't found acute toxicity reaction and allergic reaction, the chronic toxicity of carbon nanotubes to the human body need to be studied in-depth.

\section{CONCLUSIONS}

Because Nano-drug is a new type drug, the development of nano-drug will cause the revolution of the diagnosis and treatment. In recent years, nano-technology was applied in traditional Chinese medicine and birth to the new concept 'nano Chinese medicine'. Among the active ingredients of Chinese medicine, effective site, the original drug, compound and new agents that using nano-technology making has made some progress. However, at present, the basic theory of nano-technology appiled in medicine and the preparation of nano-drugs are still incomplete, especially the safety of nano- medicines has many problems remain to be explored in depth. Therefore, the research in the field of nano-technology appiled in medicine has a great deal of work needs to be done, but the superior capability that nano-drugs owns indicates a very wide range of applications in the clinical disease treatment.

\section{ACKNOWLEDGEMENTS}

This work was jointly supported by the Scientific Innovation Project of Northwest Normal University (Grant Nos: NWNU-KJCXGC-03-57, NWNU-KJCXGC-03-49) and the Proficient Talent Project of lanzhou Institute of Chemical Physics, Chinese Academy of Sciences (Grant 
No: 070430SRC1).

\section{REFERENCES}

[1] Balogh, L.P. (2009) The future of nanomedicine and the future of Nanomedicine: NBM. Nanomedicine, $\mathbf{5}, 1$.

[2] Shephard, M.J., Todd, D., Adair, B.M., Po, A.L.W., Mackie, D.P. and Scott, E.M. (2003) Immunogenicity of bovineparainfluenza type 3 virus proteins encapsulated in nanoparticle vaccines, following intranasal administration to mice. Res. Vet. Sci., 74, 187-190.

[3] Cui, Z.R.and Mumper, R.J. (2002) Intranasal administration of plasmid DNA-coated nanoparticles results in enhanced immune responses. J. Pharm. Pharmacol., 54, 1195-1203.

[4] Vijayanathan, V., Thomas, T. and Thomas, T.J. (2002) DNA nanoparticles and development of DNA delivery vehicles for gene therapy. Biochemistry, $\mathbf{4 1}$, 14085-14094.

[5] Cleland, J.L. (1998) Solvent evaporation processes for the production of controlled release biodegradable microsphere formulations for therapeutics and vaccines. Biotechnol. Prog., 14, 102-107.

[6] Esfand, R.and Tomalia, D.A. (2001) Poly (amidoamine) (PAMAM) dendrimers:from biomimicry to drug delivery and biomedical applications. Drug Discovery Today, 6, 427-436.

[7] Aukunuru, J.V., Ayalasomayajula, S.P. and Kompella, U.B. (2003) Nanoparticle formulation enhances the delivery and activity of a vascular endothelial growth factor antisense oligonucleotide in human retinal pigment epithelial cells. J. Pharm. Pharmacol., 55, 1199-1206.

[8] Maeda, H., Wu, J., Sawa, Y., Matsumura, Y. and Hori, K. (2000) Tumor vascular permeability and the EPR effect in macromolecular therapeutics:a review. J. Control. Release, 65, 271-284.

[9] Lukyanov, A.N. and Torchilin, V.P. (2004) Micelles from lipid derivatives of water-soluble polymers as delivery systems for poorly soluble drugs. Adv. Drug Delivery Rev. 56, 1273-1289.

[10] Torchilin, V.P. (2002) PEG-based micelles as carriers of contrast agents for different imaging modalities. Adv. Drug Delivery Rev., 54, 235-252.

[11] Mitra, S., Gaur, U., Ghosh, P.C. and Maitra, A.N. (2001) Tumour targeted delivery of encapsulated dextran doxorubicin conjugate using chitosan nanoparticles as carrier. J. Control. Release, 74, 317-323.

[12] Du, S.L., Pan, H., Lu, W.Y., Wang, J., Wu, J. and Wang J. Y. (2007) Cyclic Arg-Gly-Asp peptide-labeled liposomes for targeting drug therapy of hepatic fibros is in rats. $J$. Pharmacol. Exp. Ther., 322, 560-568.

[13] Briz, O., Macias, R.I.R., Vallejo, M., Silva, A., Serrano, M.A. and Marin, J.J.G. (2003) Usefulness of liposomes loaded with cytos tatic bile acid derivatives to circumvent chemotherapy res is tance of enterohepatic tumors. Mol. Pharmacol., 63, 742-750.

[14] Saul, J.M., Annapragada, A.V. and Bellamkonda, R.V. (2006) A dual-ligand approach for enhancing targeting selectivity of therapeutic nanocarriers. J. Control. Release, 114, 277-287.
[15] Hashida, M., Akamatsu, K., Nishikawa, M., Yamashita, F., Yoshikawa, H. and Takakura, Y. (2000) Design of polymeric prodrugs of PGE1 for cell-specific hepatic targeting. Pharmazie, 55, 202-205.

[16] Chung, J.E., Yokoyama, M., Aoyagi, T., Sakurai, Y. and Okano, T. (1998) Effect of molecular architecture of hydrophobically modified poly ( $\mathrm{N}$-isopropylacrylamide) on the formation of thermoresponsive core-shell micellar drug carriers. J. Control. Release, 53, 119-130.

[17] Kohori, F., Sakai, K., Aoyagi, T., Yokoyama, M., Sakurai, Y. and Okano, T. (1998) Preparation a characterization of thermally responsive block copolymer micelles comprising poly ( $\mathrm{N}$-isopropylacrylamide- $\beta$-DL-lactide). J. Control. Release, 55, 87-98.

[18] Meyer, O., Papahadjopoulos, D. and Leroux, J.C. (1998) Co- polymers of $\mathrm{N}$-isopropylacrylamide can trigger $\mathrm{pH}$ sensitivity to stable liposomes. FEBS Lett. 421, 61-64.

[19] Stover, T.C., Kim, Y.S., Lowe, T.L. and Keste, M. (2008) Thermoresponsive and bio-degradable linear-dendritic nanoparticles for targeted and sustained release of a pro-apoptotic drug. Biomaterials, 29, 359-369.

[20] Na, K., Lee, E.S. and Bae, Y.H. (2003) Adriamycin loaded pullulan acetate/sulfonamide conjugate nanoparticles responding to tumor $\mathrm{pH}$ : $\mathrm{pH}$-dependent cell interaction, internalization and cytotoxicity in vitro. J. Control. Release, 87, 3-13.

[21] Yoo, H.S., Lee, E.A. and Park, T.G. (2002) Doxorubicinconjugated biodegradable polymeric micelles having acid-cleavable linkages. J. Control. Release, 82, 17-27.

[22] Xiao S.Y., Tong, C.Y., Liu, X.M., Yu, D.M., Liu, Q.L., Xue, C.G., Tang, D.Y. and Zhao, L.J. (2006) Preparation of folate-conjugated starch nanoparticles and its application to tumor-targeted drug delivery vector. Chin. Sci. Bull, 51, 1151-1155.

[23] Pan, J. and Feng, S.S. (2008) Targeted delivery of pa clitaxel using folate-decorated poly(lactide)-vitaminE TPGS nan-oparticles. Biomaterials, 29, 2663-2672.

[24] Terada, T., Iwai, M., Kawakami, S., Yamashita, F. and Hashida, M. (2006) Novel PEG-matrix metalloproteinase-2 cleavable peptide-lipid containing galactosylated liposomes for hepatocellular carcinoma-selective targeting. J. Control. Release, 111, 333-342.

[25] Sershen, S.R., Westcoot, S.L., Halas, N.J. and West, J.L. (2000) Temperature-sensitive polymer-nanoshell composites for photothermally modulated drug delivery. $J$. Biomed. Mater. Res., 51, 293-298.

[26] Sun, Y., Lu, M. and Yin, X.F. (2006) Ntracellular delivery of fluoresent dyes mediated by nanometer-liposomes. Chem. J. Chin. U., 27, 632-634.

[27] Sivaramakrishnan, R., Nakamura, C., Mehnert, W., Korting, H.C., Kramer, K.D. and Schafer-Korting, M. (2004) Glucocorticoid entrapment into lipid carriers-characterization by parelectric spectroscopy and influence on dermal uptake. J. Control. Release, 97, 493-502.

[28] Ding, J.C., Hu, F.Q. and Yuan, H. (2004) Uptake of mono-stearin solid lipid nanoparticles by A549 cells. Acta Phamaceutica Sinica, 39, 876-880.

[29] Pantarotto, D., Partidos, C.D., Hoebeke, J., Brown, F., Kramer, E., Briand, J.P., Muller, S., Prato, M. and Bianco, A. (2003) Mmunization with peptide-functionalized carbon nanotubes enhances virus-specific neutralizing antibody responses. Chem. Biol., 10, 961-966. 
[30] Becker, M.L., Fagan, J.A., Gallant, N.D., Bauer, B.J., Bajpai, V., Hobbie, E.K., Lacerda, S.H., Migler, K.B. and Jakupciak, J.P. (2007) Length-dependent up take of DNA-wrapped single-walled carbon nanotubes. $A d v$. Mater., 19, 939-945.

[31] Ito, Y., Venkatesan, N., Hirako, N., Sugioka, N. and Takada, K. (2007) Effect of fiber length of carbon nanotubes on the absorption of erythropoietin from rat small intestine. Int. J. Pharm., 337, 357-360.

[32] Masaro, L. and Zhu, X.X. (1999) Physical models of diffusion for polymer solutions, gels and solids. Prog. Polymer Sci., 24, 731-775.

[33] Frank, A., Rath, S.K. and Venkatraman, S.S. (2005) Controlled release from bioerodible polylners : effect of drug type and polymer composition. J. Control. Release, 102, 333-344.

[34] Du, Q., Hu, J.L., Han, Y.D., Chen, X.S. and Jing, X.B. (2008) Preparation of controlled-release microspheres loading protein through solid-in-oil-water emulsion method. Chem. J. Chin. U., 29, 1262-1266.

[35] Yang, Y.N., Lou, L., Liang, Q.Z., Chen, X.S. and Jing, X.B. (2004) Preparation and in vitro release of rifampin microspheres encapsulated in biodegradable polyesters. Chem. J. Chin. U., 25, 162-165.

[36] Jain, R.A. (2000) The manufacturing techniques of various drug loaded biodegradable poly(lactide-co-glycolide) (PLGA) devices. Biomaterials, 21, 2475-2490.

[37] Wang, Y.M., Sato, H. and Horikoshi, I. (1997) In vitro and in vivo evaluation of taxol release from poly(lactic-co-glycolic acid) microspheres containing isopropyl myri- state and degradation of the microspheres. J. Control. Release, 49, 157-166.

[38] Wang, Y.S., Wang, Y.M., Li, R.S., Zhao, J. and Zhang, Q.Q. (2008) Chitosan-based self-assembled nanomicelles as a novel carrier for paclitaxel. Chem. J. Chin. U., 29, 1065- 1069.
[39] Li, J.H., Yu, Y.W., Yu, Y.L. and Shen, W. (2000) Studies on preparation of carboxymethylchitosan. Chin. J. Biochem. Pharmaceutics, 21, 175-177.

[40] Yang, X.F. and Xi, T.F. (2001) Progress in the studies on the evaluation of biocompatibility of biomaterials. $J$. Biomed. Eng., 18, 123-128.

[41] Fan, C.X. and Chen, L. (2004) Application of molecular biological methods to the study of biomaterial evaluation. Biomed. Eng.: Foreign Med. Sci., 27, 375-379.

[42] Richardson, S.C., Kolbe, H.V. and Duncan, R. (1999) Potential of low molecular mass chitosan as a DNA delivery system: biocompatibility, body distribution and ability to complex and protect DNA. Int. J. Pharm., 178, 231-243.

[43] Kim, J.S., Yoon, T.J., Yu, K.N., Kim, B.G., Park, S.J., Kim, H.W., Lee, K.H., Park, S.B., Lee, J.K. and Cho, M.H. (2006) Toxieity and tissue distribution of magnetie nanopartieles in mice. Toxicol. Sci., 89, 338-347.

[44] Yang, J.Y., Chen, Y.X. and Zhang, Y.D. (2005) In vivo distribution of silicon nanoparticles and toxicity tests, China Medical Engineering. Chin. Med. Eng., 13, 585-590.

[45] Lam, C.W., Jmes, J.T., McCluskey, R. and Hunter, R.L. (2004) Pulmonary toxicity of single-wall carbon nanotubes in mice 7 and 90 days after intratracheal instillation. Toxicol. Sci., 77, 126-134.

[46] Service, R.F. (2003) Nanomaterials show signs of toxicity. Science, 300, 243.

[47] Yang, S.T., Guo, W., Lin, Y., Deng, X. Y., Wang, H. F., Sun, H.F., Liu, Y.F., Wang, X., Wang, W., Chen, M. Huang, Y.P. and Sun, Y.P. (2007) Biodistribution of pristine single-walled carbon nanotubes in vivo. J. Phys. Chem. C, 111, 17761-17764. 